\title{
Catastrophic Antiphospholipid Syndrome Associated with Systemic Lupus Erythematosus Successfully Treated with Rituximab: A Case Report
}

\author{
JinShik Shin, Kwang Nam Kim \\ Department of Pediatrics, Hallym University Sacred Heart Hospital, Anyang, Korea
}

The catastrophic variant of antiphospholipid syndrome (APS) is a very rare and life-threatening condition of APS. This condition is characterized by thrombosis in multiple organs within a short period of time in the presence of positive antiphospholipid antibodies (aPL). Over the past few decades, considerable progress has been made in the treatment of patients with catastrophic APS; however, the mortality rate still remains very high. Although some cases of rituximab treatment in patients with catastrophic APS have been reported, there is no clear treatment protocol. A 14-year-old girl with systemic lupus erythematosus was diagnosed with catastrophic APS. She received several medications: corticosteroids, intravenous immunoglobulin, and plasmapheresis with anticoagulants. Unfortunately, she did not improve, and rituximab was started with four courses. After the rituximab treatment, she did not experience further thrombotic events during the follow up. This paper reports a pediatric case of catastrophic APS treated successfully with rituximab in Korea. (J Rheum Dis 2019;26:74-78)

Key Words. Antiphospholipid syndrome, Systemic lupus erythematosus, Rituximab

\section{INTRODUCTION}

Antiphospholipid syndrome (APS) is a multisystem autoimmune disease characterized by arterial thrombosis, venous thrombosis associated with persistently positive antiphospholipid antibodies (aPL) [1]. aPL comprises three main antibodies: lupus anticoagulant (LA), anticardiolipin, and anti-beta2-glycoprotein I (anti- $\beta$ 2-GPI). APS may occur alone or in conjunction with systemic lupus erythematosus (SLE) or rheumatoid arthritis (RA) [2]. However, the pathophysiological mechanisms of this correlation are unclear [3].

Catastrophic APS is a rare, severe, and life-threatening form of APS resulting in multiorgan failure and affects approximately less than $1 \%$ of patients with APS [4]. Furthermore, the low prevalence of pediatric catastrophic APS makes its diagnosis difficult. Therefore, early diagnosis and aggressive treatments are very important. The preliminary criteria for the classification of catastrophic APS were presented by the International Task Force in 2003 [4], and were ultimately validated by an extended report published in 2005 [5]. These criteria help clinicians recognize circulating aPL and the rapid onset of thrombosis in multiple organs (Table 1) [5].

Because of currently available conventional combined treatment regimens, such as anticoagulants, corticosteroids, plasmapheresis, and immunoglobulins (IVIGs), the mortality rates have decreased from $53 \%$ to $37 \%$, compared before 2001 [1,6]. Despite the significant decrease, the mortality rate is still high and there is no definite effective treatment. Recently, rituximab (Mabthera) has been shown to be effective as a treatment for catastrophic APS. However, there are few studies on the use of rituximab in treating catastrophic APS in both adults and children.

We herein report the case of a 14-year-old girl with cata-

Received : April 5, 2018, Revised : May 10, 2018, Accepted : May 28, 2018

Corresponding to : Kwang Nam Kim (D) http://orcid.org/0000-0003-4024-5128

Department of Pediatrics, Hallym University Sacred Heart Hospital, 22 Gwanpyeong-ro 170beon-gil, Dongan-gu, Anyang 14068, Korea. E-mail : kwangnamkim@naver.com 
Table 1. Preliminary criteria for the classification of catastrophic antiphospholipid syndrome (APS)

1) Evidence of involvement of three or more organs, systems and/or tissues*

2 Development of manifestations simultaneously or in less than a week ${ }^{\dagger}$

3) Confirmation by histopathology of small vessel occlusion in at least one organ or tissue

4) Laboratory confirmation of the presence of antiphospholipid antibodies (lupus anticoagulant and/or anticardiolipin antibodies) ${ }^{\ddagger}$

Definite catastrophic APS

- All four criteria

Probable catastrophic APS

- All four criteria, except for only two organs, systems and/or tissues involvement

- All four criteria, except for the absence of laboratory confirmation at least 6 weeks apart due to the early death of a patient never tested for antiphospholipid antibodies before the catastrophic APS

- 1,2 and 4

- 1, 3 and 4 and the development of a third event in more than a week but less than a month, despite anticoagulation

*Usually, clinical evidence of vessel occlusions, confirmed by imaging techniques when appropriate. Renal involvement is defined by a $50 \%$ rise in serum creatinine, severe systemic hypertension $(>180 / 100 \mathrm{mmHg})$ and/or proteinuria $(>500$ $\mathrm{mg} / 24$ hours). ${ }^{\dagger}$ For histopathological confirmation, significant evidence of thrombosis must be present, although vasculitis may coexist occasionally. ${ }^{\ddagger}$ If the patient had not been previously diagnosed as having an APS, the laboratory confirmation requires that presence of antiphospholipid antibodies must be detected on two or more occasions at least six weeks apart (not necessarily at the time of the event). Reproduced from Asherson et al. Lupus 2003;12:530-4, with permission of SAGE Publications [4].

strophic APS who was successfully treated with rituximab.

\section{CASE REPORT}

A 14-year-old girl was admitted with right fifth toe's pain and necrotic changes. She was diagnosed with SLE at the age of 11 and was continuously treated with hydroxychloroquine, prednisolone (PD) and azathioprine. At that time, she was in the process of tapering the dose of PD, and was taking it at a dose of $5 \mathrm{mg}$ twice a day, just before admission. Three months before admission, she experienced acute pain with skin color change in right fifth toe, so she received with aspirin treatment (100 mg daily) additionally. At the time of admission, she had no fever

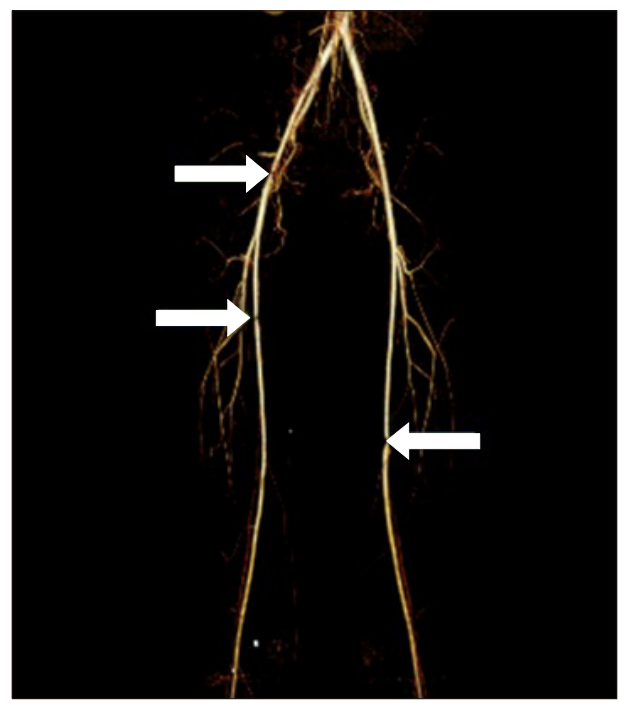

Figure 1. Contrast enhanced computed tomography angiography of lower extremity: multifocal stenosis at right distal common iliac artery, right upper superficial femoral artery, and left lower superficial femoral artery (white arrows).

and her vital signs including blood pressure (BP) were stable. Laboratory results showed hemolytic anemia (hemoglobin [Hb] $6.6 \mathrm{~g} / \mathrm{dL}$ ), positive Direct/indirect Coombs' test, prolonged activated partial thromboplastin time (aPTT) (99.5 seconds), increased total bilirubin $(2.38 \mathrm{mg} / \mathrm{dL}$ ) (particularly indirect bilirubin $1.79 \mathrm{mg} / \mathrm{dL}$ ), and disseminated intravascular coagulation (DIC) profiles such as fibrin degradation product (FDP), D-dimer, fibrinogen levels were within normal range. She tested positive for anti-dsDNA, anti-nuclear antibodies, anticardiolipin IgM and IgG antibodies, anti- $\beta 2$-GPI and LA. Ultrasonography (US) and computed tomography (CT) angiography of the lower extremity showed multiple stenoses in the right distal common iliac artery, right upper superficial femoral artery, and left lower superficial femoral artery (Figure 1). Despite the treatment, the symptoms did not resolve, and necrotic changes gradually worsened on the right fifth toe. Therefore, percutaneous transluminal angioplasty was performed, and low-molecular-weight heparin (LMWH) was added to the medication. However, autoamputation of the right fifth toe occurred six months later.

Three months later, she was again admitted for vomiting, diarrhea, and abdominal pain without fever. On initial examination, her vital signs were stable and there were no clinical signs of acute peritonitis. Laboratory results showed hemolytic anemia ( $\mathrm{Hb} 5.6 \mathrm{~g} / \mathrm{dL}$ ), thrombocytopenia (platelet count, $40 \times 10^{3} / \mu \mathrm{L}$ ), elevated C-re- 
active protein $(93.4 \mathrm{mg} / \mathrm{L}$ [normal, $0.0 \sim 5.0 \mathrm{mg} / \mathrm{dL}$ ]), and prolonged aPTT (99.5 seconds). Fibrinogen (683 $\mathrm{mg} / \mathrm{dL})$, FDP $(7.91 \mu \mathrm{g} / \mathrm{mL})$, D-dimer $(1.93 \mu \mathrm{g} / \mathrm{mL})$, and antithrombin III (128\%) were elevated. She tested positive for LA, anticardiolipin IgM and IgG antibodies, and anti- $\beta 2$-GPI. Abdominal US and CT revealed hepatosplenomegaly, long and segmental jejunal wall thickening with a large amount of ascites (Figure $2 \mathrm{~A} \sim \mathrm{C}$ ). Thus, intravenous (IV) methylprednisolone (MPD) pulse therapy ( $1 \mathrm{~g} /$ day for 3 days) was initiated under the diagnosis of lupus enteritis. The day after initiation of MPD pulse therapy, shortness of breath, palpitation, and retrosternal pain suddenly occurred. On electrocardiography, ST-segment elevation was noted at lead II, III, and aVF, and 0.6-mg sublingual nitroglycerin was administered (Figure 3). Emergency laboratory tests showed elevated cardiac enzyme levels (creatine kinase [CK], 1,291 IU/L; CK-MB, $170.9 \mathrm{ng} / \mathrm{mL}$; and troponin I, $16.15 \mathrm{ng} / \mathrm{mL}$ ). Due to acute myocardial infarction (MI), emergency coronary angiography was performed, and minimal lesions were observed in the left circumflex coronary artery and the left anterior descending artery. On chest CT, low-attenuation lesions considered as ischemic changes around the left ventricle were observed with a large amount of pleural fluid (Figure 4). An Echocardiogram showed hypofunction of the right ventricle, and myocarditis was strongly suspected. On the eighth day of admission, she complained of chest and back pain with fever (body temperature of $\geq 38.5^{\circ} \mathrm{C}$ ) and was identified as having reduced BP 80/40 mmHg. Thus, we administered inotropics, such as dopamine and dobutamine with analgesics. Thereafter, her symptoms and vital signs gradually improved, and MPD pulse therapy was restarted. With treatment, her symptoms subsided and platelet count also normalized.

One month later, she was admitted for the third time; this time, the presented with petechial lesions of the limbs and oral mucosal bleeding. Laboratory tests showed thrombocytopenia (platelet count, $6 \times 10^{3} / \mu \mathrm{L}$ ) with prolonged aPTT (99.3 seconds) and DIC profiles were within normal range. The clinical course did not improve despite IVIG administration for 5 days at a dose of $2 \mathrm{~g} / \mathrm{kg}$ once a day and transfusions. Thus, seven rounds of

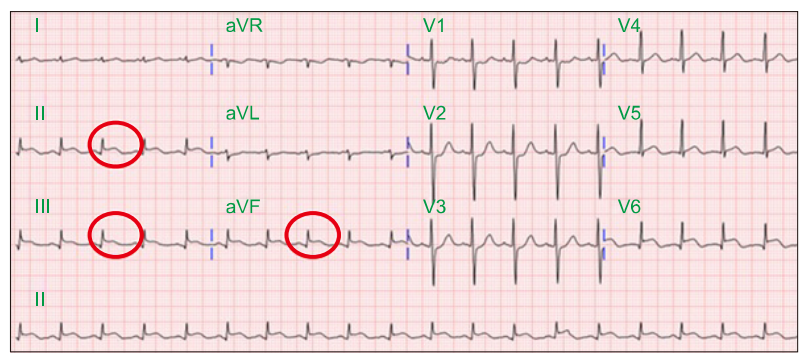

Figure 3. Electrocardiography: ST elevation in lead II, III, aVF.

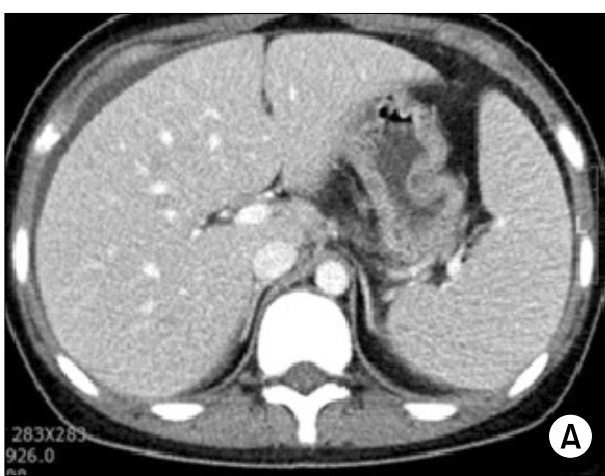

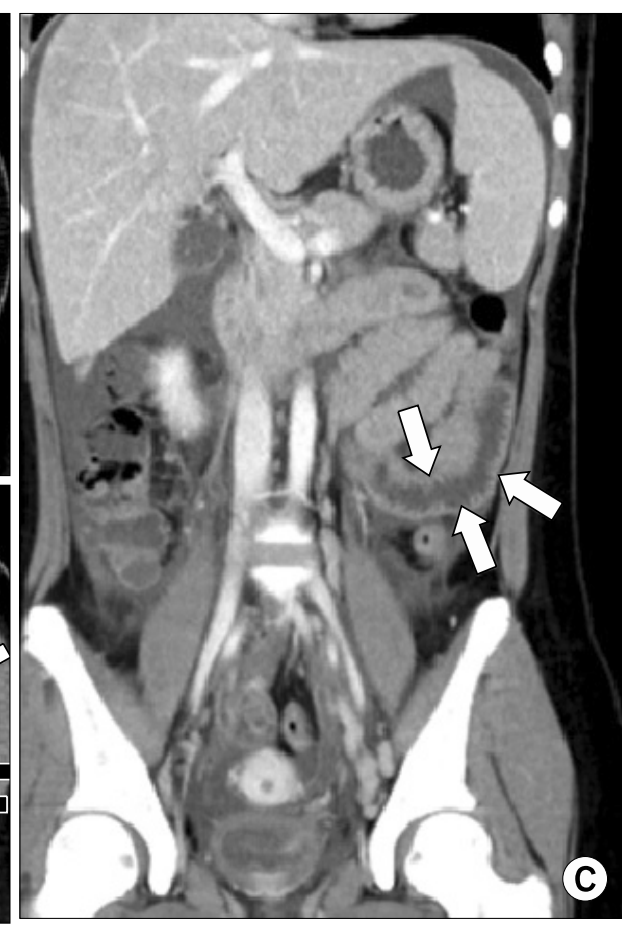

Figure 2. Contrast enhanced computed tomography of abdomen. (A) Hepatosplenomegaly. (B, C) Long segmental jejunal wall thickening (white arrows) with a large amount of ascites (black arrows). 


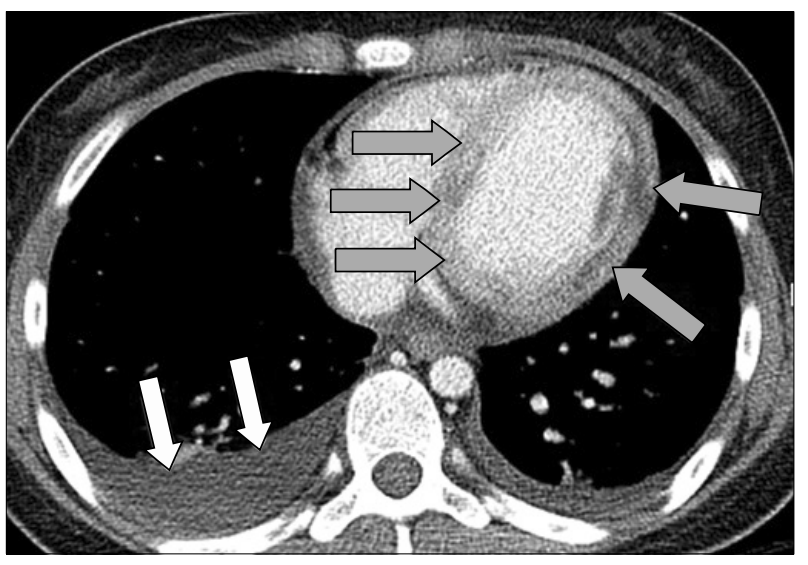

Figure 4. Contrast enhanced computed tomography of chest. Low attenuation lesions considered as ischemic changes around the left ventricle (gray arrows) with a large amount of pleural fluid (white arrows).

plasmapheresis were administered. After plasmapheresis, the platelet count increased to $57 \times 10^{3} / \mu \mathrm{L}$; however, the effect was transient. Despite plasmapheresis, her condition showed no resolution, and ultimately, we decided to administer rituximab. Rituximab was administered at a dose of $375 \mathrm{mg} / \mathrm{m}^{2}$ by IV infusion once weekly for 4 weeks. The skin lesions were resolved. Blood tests revealed that the platelet count increased to $99 \times 10^{3} / \mu \mathrm{L}$ two weeks after her discharge and returned to the normal range a month later. The patient is being followed up regularly at our outpatient rheumatologic clinic and shows no recurrence of skin lesions or any other organ involvement for three years. In a recent blood test for aPL, LA was positive, and anticardiolipin and anti$\beta$ 2-GPI were negative.

\section{DISCUSSION}

We reported the case of a 14-year-old girl with catastrophic APS. She was diagnosed with catastrophic APS with SLE on the basis of skin necrosis, small artery thromboses of lower extremities, lupus enteritis, acute MI, acute myocarditis, and positive aPL. The patient was confirmed as aPL-positive at least twice, 10 weeks apart. She was treated with corticosteroids, IVIGs, plasmapheresis, and anticoagulants. However, there was no improvement, and therefore, rituximab was administered. After rituximab treatment, no further thrombotic events have occurred during last 3 years of follow-up.

Currently, there are no standard recommendations or guidelines for the treatment of catastrophic APS because it is a very rare disease in both adults and children [7]. Anticoagulation therapy is the first-line and the most important step in the treatment of catastrophic APS. Antiplatelet therapy can be used in addition to anticoagulant therapy, particularly in patients with arterial thrombotic events [8]. According to the 8th American College of Chest Physicians (ACCP) guidelines, therapeutic and prophylactic uses of $\mathrm{LMWH}$ are based on the anti-factor Xa assay (anti-FXa) ranges of $0.5 \sim 1.0 \mathrm{U} / \mathrm{mL}$ and $0.1 \sim 0.3 \mathrm{u} / \mathrm{mL}$, respectively. The ACCP guidelines suggest prophylactic doses of $0.75 \mathrm{mg} / \mathrm{kg}$ and $0.5 \mathrm{mg} / \mathrm{kg}$ every 12 hours for neonates $<2$ months and children older than 2 months, respectively, to bring anti-FXa levels in the therapeutic range. In addition, the guidelines for treatment of thrombosis with enoxaparin are based on a study which recommended a dose of $1.5 \mathrm{mg} / \mathrm{kg}$ every 12 hours for neonates $<2$ months and $1.0 \mathrm{mg} / \mathrm{kg}$ every 12 hours for children older than 2 months [9]. LMWH at a dose of 1.0 $\mathrm{mg} / \mathrm{kg}$ was administered every 12 hours to our patient in the present study. There is a linear relationship between the anti-FXa activity and aPTT with a good correlation, and thus, aPTT may be suitable for monitoring thrombosis prophylaxis in patients at high risk of accumulation of LMWH [10]. We are accordingly adjusting the dose of LMWH through the aPTT level. Second-line treatments include plasmapheresis and/or immunomodulatory therapies. In the present study, we performed seven rounds of plasmapheresis during the third admission. Despite these second-line therapy measures, no clinical or laboratory improvements were seen in our patient. This indicated the possibility of refractory catastrophic APS. Rituximab has recently been proven effective in treating catastrophic APS in patients unresponsive to conventional treatment $[11,12]$. In our patient in the present study, rituximab was administered because there was no improvement despite first- and second-line treatments. Rituximab administration caused a dramatic effect in the course of the disease, stopping the inflammatory process. Thereafter, all clinical and laboratory findings showed improvements, and remission is being maintained.

Based on our and previous studies, rituximab may be considered as a treatment for SLE [13]. However, further study is needed to elucidate therapeutic effect and safety data of rituximab on catastrophic APS associated with SLE.

\section{SUMMARY}

Catastrophic APS is a rare, severe, and life-threatening 
condition with a high mortality rate. Thus, early recognition and aggressive therapy are very important for successful treatment. This study is the first report of pediatric catastrophic APS treated with rituximab in Korea. Based on the recent data from several case reports considering the use of rituximab in aPL-positive patients, and results from the present review, rituximab has been used successfully in the treatment of aPL-positive patients, particularly those with refractory catastrophic APS.

\section{CONFLICT OF INTEREST}

No potential conflict of interest relevant to this article was reported.

\section{REFERENCES}

1. Rodríguez-Pintó I, Moitinho M, Santacreu I, Shoenfeld Y, Erkan D, Espinosa G, et al. Catastrophic antiphospholipid syndrome (CAPS): Descriptive analysis of 500 patients from the International CAPS Registry. Autoimmun Rev 2016;15:1120-4.

2. Erre GL, Pardini S, Faedda R, Passiu G. Effect of rituximab on clinical and laboratory features of APS; a case report and a review of literature. Lupus 2008;17:50-5.

3. Whitaker KL. Antiphospholipid antibody syndrome: The difficulties of diagnosis. JAAPA 2017;30:10-4.

4. Asherson RA, Cervera R, de Groot PG, Erkan D, Boffa MC, Piette JC, et al. Catastrophic antiphospholipid syndrome: international consensus statement on classification criteria and treatment guidelines. Lupus 2003;12:530-4.
5. Cervera R, Font J, Gómez-Puerta JA, Espinosa G, Cucho M, Bucciarelli S, et al. Validation of the preliminary criteria for the classification of catastrophic antiphospholipid syndrome. Ann Rheum Dis 2005;64:1205-9.

6. Bucciarelli S, Espinosa G, Cervera R, Erkan D, GómezPuerta JA, Ramos-Casals M, et al. Mortality in the catastrophic antiphospholipid syndrome: causes of death and prognostic factors in a series of 250 patients. Arthritis Rheum 2006;54:2568-76.

7. Sukara G, Baresic M, Sentic M, Brcic L, Anic B. Catastrophic antiphospholipid syndrome associated with systemic lupus erythematosus treated with rituximab: case report and a review of the literature. Acta Reumatol Port 2015;40:169-75.

8. Ortel TL, Erkan D, Kitchens CS. How I treat catastrophic thrombotic syndromes. Blood 2015;126:1285-93.

9. Monagle P, Chalmers E, Chan A, deVeber G, Kirkham F, Massicotte P, et al. Antithrombotic therapy in neonates and children: American College of Chest Physicians EvidenceBased Clinical Practice Guidelines (8th Edition). Chest 2008;133(6 Suppl):887S-968S.

10. Thomas O, Lybeck E, Strandberg K, Tynngård N, Schött U. Monitoring low molecular weight heparins at therapeutic levels: dose-responses of, and correlations and differences between aPTT, anti-factor Xa and thrombin generation assays. PLoS One 2015;10:e0116835.

11. Doğru A, Ugan Y, Şahin M, Karahan N, Tunç ŞE. Catastrophic antiphospholipid syndrome treated with rituximab: A case report. Eur J Rheumatol 2017;4:145-7.

12. Haskin O, Amir J, Schwarz M, Schonfeld T, Nahum E, Ling $\mathrm{G}$, et al. Severe abdominal pain as a presenting symptom of probable catastrophic antiphospholipid syndrome. Pediatrics 2012;130:e230-5.

13. Mok CC. Current role of rituximab in systemic lupus erythematosus. Int J Rheum Dis 2015;18:154-63. 